\title{
Results and Patient Reported Outcome Measures (PROMs) after One-Stage Revision for Periprosthetic Joint Infection of the Hip: A Single-centre Retrospective Study
}

\author{
Jesse W.P. Kuiper ${ }^{1,2^{\star 凶}}$, Christine M.E. Rustenburg ${ }^{1}$, Jore H. Willems ${ }^{3,2^{*}}$, Steven J. Verberne' ${ }^{2}$, Edgar J.G. \\ Peters 4,5 , Rachid Saouti ${ }^{1}$ \\ 1. Department of Orthopaedic Surgery, VU University Medical Centre, Amsterdam, the Netherlands \\ 2. Department of Orthopaedic Surgery, Noord-West Ziekenhuisgroep, Alkmaar, the Netherlands \\ 3. Department of Orthopaedic Surgery, Spaarne Gasthuis, Hoofddorp, the Netherlands \\ 4. Department of Internal Medicine, VU University Medical Centre, Amsterdam, the Netherlands \\ 5. Amsterdam Infection and Immunity Institute, Amsterdam, the Netherlands \\ *: present address \\ $\square$ Corresponding author: J.W.P. Kuiper, jwp.kuiper@gmail.com, Noord-West Ziekenhuisgroep Alkmaar, Wilhelminalaan 12, 1815 JD, Alkmaar, the \\ Netherlands, 0031-72-5482500 \\ (c) Ivyspring International Publisher. This is an open access article distributed under the terms of the Creative Commons Attribution (CC BY-NC) license \\ (https://creativecommons.org/licenses/by-nc/4.0/). See http://ivyspring.com/terms for full terms and conditions.
}

Received: 2017.12.14; Accepted: 2018.06.13; Published: 2018.07.06

\begin{abstract}
Background: Little is known about functional outcome and quality of life (QoL) after one-stage revision for periprosthetic joint infection (PJI) of the hip.

Methods: a cohort of 30 subjects treated with one-stage revision between 2011 and 2015 was identified, and questionnaires on functional outcome and QoL were distributed.

Results: 28 subjects were successfully treated (93\%). Most subjects were referred from other hospitals. Coagulase-negative Staphylococcus was found in $50 \%$ of the cases, and $40 \%$ of all cultured bacteria were multidrug-resistant. $25 \%$ had subsequent revision surgery, unrelated to PJI. Functional outcome was good and QoL scores were high, comparable to prosthetic joint revision surgery in general.

Conclusion: Although the cohort was small and statistical analysis was not performed, this study showed that excellent results can be obtained with one-stage revision for hip PJI. Functional outcome and QoL was comparable to prosthetic joint revision surgery in general.
\end{abstract}

Key words: one-stage revision; periprosthetic joint infection; hip; outcome; functional outcome; quality of life

\section{Introduction}

Periprosthetic joint infection (PJI) of the hip is one of the most precarious complications of total hip arthroplasty (THA). It generally requires one or more operations, weeks of hospitalization and long courses of antibiotic treatment. It is a great financial and logistic burden to hospitals and health care in general[1,2]. The patients themselves, however, are the ones most afflicted by the complication. Treatment methods range from life-long suppressive antibiotic therapy (for inoperable patients with a low grade PJI) to months of living without a functioning hip articulation (Girdlestone procedure) and to curative therapy with joint replacement[2]. In joint replacement therapy, two-stage revision is the gold standard. In this procedure, the arthroplasty is resected and reimplantation is performed after weeks 
or months of treatment with antibiotics. A one-stage revision, however, according to guidelines, is the preferred option for non-acute PJI in patients with an adequate soft tissue envelope, sufficient bone stock and a pre-operatively identified non-resistant micro-organism[2].

In one-stage revision, all arthroplasty components are removed, including any poly-methylmethacrylate (PMMA) bone cement, thorough surgical debridement and extensive irrigation are performed, after which new arthroplasty components are directly reimplanted. Rigorous removal of all possibly contaminated tissue and foreign body material is paramount for infection eradication[2,3]. Success rates of one-stage revision in different studies vary between 76 and $100 \%$, but in patients with favourable circumstances (e.g. infection with non-resistant micro-organism, adequate soft tissue) lie around $90 \%[2,4]$. A recent systematic review suggested that one-stage revision may be comparable to two-stage revision in terms of reinfection risk[3].

Studies on functional outcomes after one-stage revision are scarce, and only one found better functional outcomes compared to two-stage revision (Harris Hip Score and Visual Analogue Scale for pain)[5]. To our knowledge, no published studies have described the effect of one-stage revision on the quality of life (QoL) of patients[6]. However, a trial protocol has been published on one-stage versus two-stage revisions, including QoL and functional outcome at follow-up, but these results have not been published yet[7].

The aim of this study is to describe the outcomes of a retrospectively selected cohort after one-stage revision for hip PJI, in terms of reinfection rate, functional scores and quality of life.

\section{Material and methods}

\section{Subject selection and inclusion}

We searched our hospital's database of performed surgical procedures for all ICPC (International Classification of Primary Care) and surgery codes possibly linked to PJI procedures, to find patients who were surgically treated for hip PJI between January 2011 and December 2015. Electronic patient records were retrospectively analysed and relevant data were extracted. Subjects were included if they underwent one-stage revision for PJI of THA in the study period. Excluded were subjects with PJI of a hemi-arthroplasty, incomplete removal of foreign body material (i.e. arthroplasty components or bone cement) and subjects in whom PJI criteria were not met. PJI criteria were: two or more positive periprosthetic cultures with phenotypically identical organisms, or a sinus tract communicating with the joint, or having at least three of the following minor criteria: elevated serum C-reactive protein (CRP) AND erythrocyte sedimentation rate (ESR); elevated synovial fluid white blood cell (WBC) count OR ++ result on leukocyte esterase test strip; elevated synovial fluid polymorphonuclear neutrophil percentage (PMN\%); positive histological analysis of periprosthetic tissue; a single positive culture[8]. If subjects died within a year of follow-up, unrelated to PJI, success was defined as 'uncertain', and subjects were excluded. A minimal follow-up of one year was required in all other cases.

\section{Informed consent and ethical approval}

Ethical approval for this study was received from the ethical committee of our hospital, with approval number 2017.181. Data collection and subject contacts were handled according to the ethical standards in the 1964 Declaration of Helsinki.

Subjects meeting our inclusion criteria were informed about the proposed study by letter and invited to participate. Instructions and information about the study were included, along with the questionnaires and reply envelope. If no reply was received, subjects were contacted by telephone.

\section{Treatment}

In our institution, one-stage revision for PJI is performed by one orthopaedic surgeon, the senior author (RS), and is the treatment method for all patients with hip PJI for chronic infection or when a sinus tract is present. Two-stage revision is only performed when patients have sepsis or are otherwise severely immunocompromised (e.g. undergoing chemotherapy treatment), or when bone defects are so extensive that a tumour prosthesis is necessary. This is a protocol that is not generally performed in other Dutch hospitals, where one-stage revision is much less performed.

All hips were revised using a posterolateral approach in lateral decubitus position. Surgery was performed under strict sterile conditions. After removal of all arthroplasty components, complete PMMA bone cement removal (if present), thorough debridement and irrigation with at least $6 \mathrm{~L}$ of saline, reimplantation was performed in the same session. Choice of THA model and use of (gentamicin loaded) bone cement was based on bone stock and quality, whether a fracture had occurred and whether osteotomy was required for THA removal. In most cases an uncemented primary or modular stem was used, and the acetabular component was also uncemented in the majority of cases. If subjects were not already receiving antibiotics 
aimed at pathogens from culture results prior to surgery, they received intravenous vancomycin (1000 milligrams twice daily, adjusted to renal function and serum levels) and ciprofloxacin (400 milligrams three times daily, or adjusted to renal function) after all cultures were taken. Postoperative antibiotic therapy, based on bacterial susceptibility (according to the European Committee on Antimicrobial Susceptibility Testing (EUCAST) definitions) and in consultation with an infectious diseases specialist and a medical microbiologist, was administered for a minimum of 6 weeks, the standard duration being 3 months, but ultimate therapy duration depended on an individual subject's parameters e.g. presence of clinical and laboratory signs of inflammation and tolerance of therapy. Intravenous therapy was usually given for two weeks, followed by oral antibiotic therapy when agents with a high bioavailability were available e.g. rifampicin and quinolones. In subjects where such antibiotics could not be given, due to inherent or acquired antimicrobial resistance or allergies, outpatient parenteral antibiotic therapy was given.

\section{Outcome}

Successful outcome was defined as retainment of THA after one-stage revision, without any subsequent surgical procedures for PJI (debridement, antibiotics, irrigation, and retention (DAIR), one-stage or two-stage revision or arthroplasty removal), without the chronic use of antimicrobial agents, and without signs or symptoms of PJI at follow-up. If other surgical procedures were performed, patient records were checked: if the surgical report, postoperative notes or culture results suggested PJI relapse, this was defined as treatment failure. Otherwise, this was noted as 'revision for other reason', and treatment was regarded successful. In patient records, culture results were checked and infections were classified as whether or not being polymicrobial PJI and culture negative PJI, and micro-organisms were categorized as non-resistant or multidrug-resistant according to standard definitions[9]. If subjects died as a result of PJI, this was defined as failure. As mentioned above, if subjects died within a year of follow-up, unrelated to PJI, success was defined as 'uncertain', and subjects were excluded. If subjects died after more than a year of follow-up, unrelated to PJI, and without subsequent procedures related to PJI, treatment was considered successful.

\section{Questionnaires}

For measurement of outcome, three questionnaires were used, according to the advice of the Dutch Orthopaedic Society (Nederlandse Orthopaedische Vereniging, NOV)[10]: Oxford Hip
Score (OHS, score range 0 to 48 ), Hip disability and Osteoarthritis Outcome Score-Physical function Short-form (HOOS-PS, score range 0-100) and EuroQol five dimensions questionnaire (EQ-5D, score range -0.329 to 1$)$. All three have validated Dutch translations[11-13]. Furthermore, Visual Analogue Score (VAS) pain for current pain (100-0), VAS satisfaction for overall satisfaction after one-stage revision (0-100) and a question whether the subject had been treated elsewhere for the same hip after the one-stage revision, were added.

\section{Results}

In the study period, 79 subjects were treated for PJI of the hip. 1 subject died within a year (after 3 weeks, unrelated to PJI), and treatment success was therefore classified as 'uncertain'. This subject was removed from further analysis. Of the remaining subjects, 30 met the inclusion criteria (Figure 1). Of these 30, 2 subjects underwent subsequent surgical procedures related to PJI after one-stage revision, and were therefore classified as treatment failure (1 subject underwent DAIR twice after one-stage revision, and 1 subject underwent DAIR and a subsequent Girdlestone procedure after one-stage revision). 28 Subjects were treated successfully $(93 \%)$. Because only 2 cases were categorized as treatment failure, no statistical analysis was performed on the data.

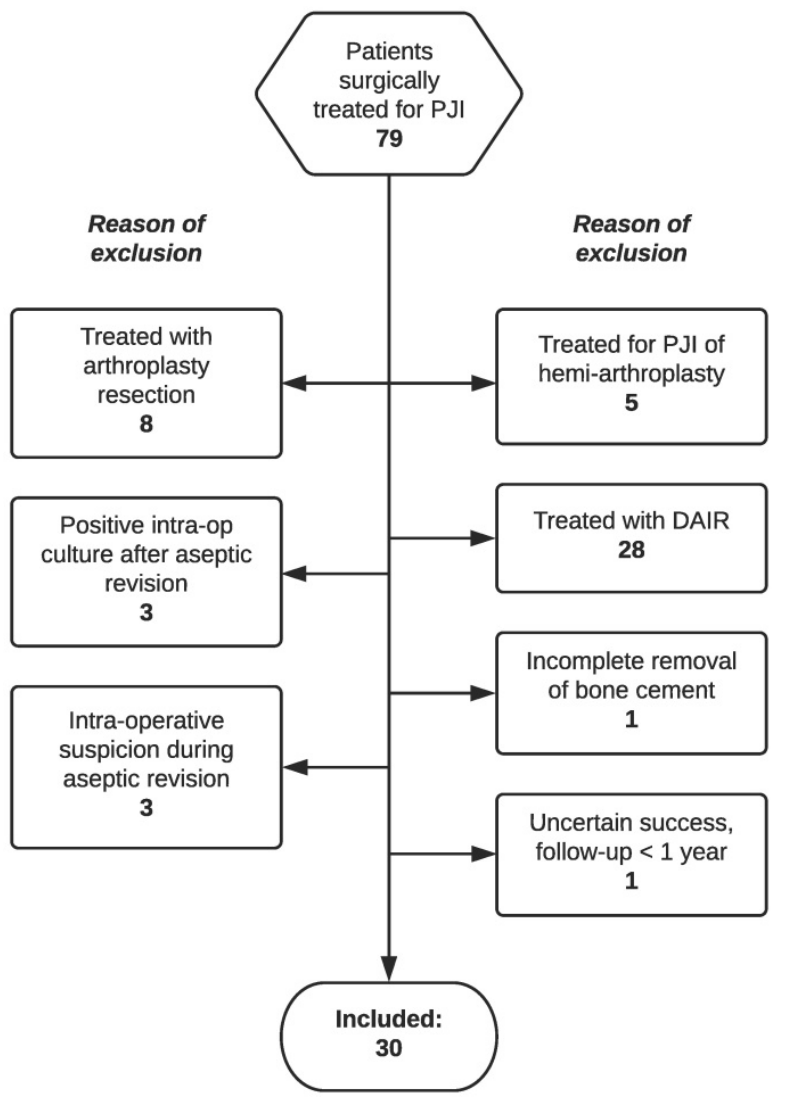

Figure 1: Flowchart of subject inclusion with numbers. 
All patients received 6-12 weeks of antibiotic treatment. The mean follow-up was more than 3 years. During the follow-up, 5 subjects died, of which 1 in the failure group (unrelated to PJI, but underwent two subsequent DAIR procedures). For subject characteristics, see Table 1 . For a list of subjects and their positive PJI criteria, see Table 2.

Most subjects were patients referred from other hospitals $(60 \%)$. A sinus tract was present in $37 \%$ of all subjects. In 23 cases, the causative micro-organism was known preoperatively $(77 \%)$, either from aspiration or positive blood culture results. In $50 \%$ of all cases, coagulase-negative Staphylococci were found. $40 \%$ of all cultured bacteria were multidrug-resistant to antibiotic agents (all in the failure group). None of the multidrug-resistant micro-organisms were resistant to vancomycin, when vancomycin susceptibility was tested. No methicillin resistant Staphylococcus aureus (MRSA) was cultured. $23 \%$ subjects had a polymicrobial PJI. In 2 cases $(6 \%)$, cultures remained negative.
Table 1: Subject characteristics.

\begin{tabular}{llll}
\hline & TOTAL & SUCCESS & FAILURE \\
\hline Number of subjects & 30 & 28 & 2 \\
Mean age in years (SD) & $71.8(10.2)$ & $71.0(10.1)$ & $82.1(6.1)$ \\
Mean follow-up in months (SD) & $39.8(19.8)$ & $41.3(19.3)$ & $18.7(17.7)$ \\
Male: female (\% male) & $9: 21(43)$ & $9: 19(47)$ & $0: 2(0)$ \\
Deceased (\%) & $5(17)$ & $4(14)$ & 1 \\
ASA classification & & & \\
I & 4 & 4 & 0 \\
II & 11 & 11 & 0 \\
III & 14 & 12 & 2 \\
IV & 1 & 1 & 0 \\
V & 0 & 0 & 0 \\
Current smoker (\%) & $6(20)$ & $6(21)$ & 0 \\
Body mass index (SD) & $28.8(6.4)$ & $28.8(6.0)$ & $29.0(14.3)$ \\
Body mass index > 30 kg/m2 (\%) & $11(37)$ & $10(36)$ & 1 ** \\
Body mass index > 35 kg/m2 (\%) & $6(20)$ & $5(18)$ & $1^{* *}$ \\
Co-morbidities* & & & \\
Cardiovascular (\%) & $18(60)$ & $17(61)$ & 1 \\
Pulmonary (\%) & $8(27)$ & $8(29)$ & 0 \\
Renal failure (\%) & 2 (7) & $2(7)$ & 0 \\
Diabetes mellitus (\%) & $3(10)$ & $3(11)$ & 0 \\
\hline ASA = American Society of Anesthesiologists Score; SD = standard deviation; \\
*Subjects were considered to have a comorbidity if it was described in the subject's \\
medical records or if the subject used medication for the comorbidity. **Both are the \\
same patient, with a body mass index of 39. & &
\end{tabular}

Table 2: Case characteristics and criteria for confirmation of PJI, according to definition.

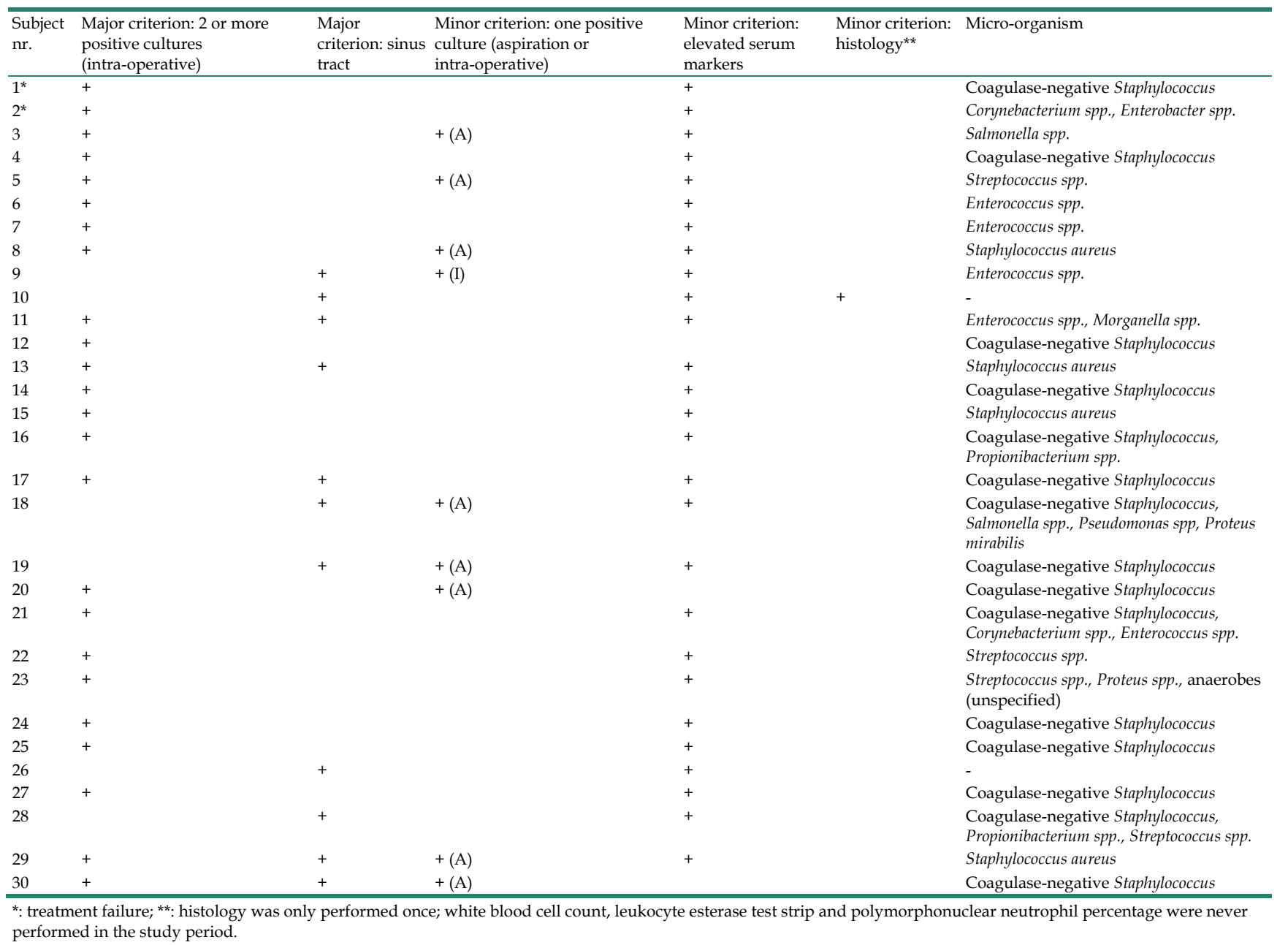


The average duration of surgery was 182 minutes. Bone allograft was used in 3 cases $(10 \%)$, of which 2 cases of failure. Femoral osteotomy was performed in $40 \% \quad(n=12)$, and intra-operative fractures occurred in $13 \%(n=4)$. For subjects with treatment failure, surgery time was longer than for subjects with treatment success, as well as more frequent use of allograft bone, more frequently performed femoral osteotomy and occurrence of fractures. Reoperations unrelated to PJI were performed in $25 \%$ of successfully treated subjects $(n=7)$ : of these, revision for recurrent dislocations took place in 4 cases, 1 subject was treated for a periprosthetic fracture three months after one-stage revision, removal of osteosynthesis material in 1 case, and femoral stem revision after stem subsidence occurred in 1 subject. In all cases, cultures in subsequent procedures were negative.

21 subjects responded to our survey $(84 \%$ of all living subjects), but some replied only partly. Survey results can be seen in Table 3 . For successfully treated subjects, the mean HOOS was 63 (of 100), OHS 35 (of 48), EQ-5D 0.68 (of 1), EQ-5D-VAS 74 (of 100), VAS pain 31 (of 100), and VAS satisfaction 81 (of 100). The only living subject with treatment failure had worse functional and QoL scores, except for VAS pain (0). None of the respondents reported subsequent surgery on the affected hip elsewhere.

Table 3: Survey results; worst and best scores respectively: HOOS 0-100; OHS 0-48, EQ-5D 0-1, EQ-5D-VAS 0-100, VAS pain 100-0, VAS satisfaction 0-100.

\begin{tabular}{llll}
\hline & TOTAL & SUCCESS & FAILURE \\
\hline HOOS & $64($ SD 28, range 15-100, $\mathrm{n}=21)$ & $63($ SD 29, $\mathrm{n}=20)$ & $90(\mathrm{n}=1)$ \\
OHS & $35($ SD 11, range 13-48, $\mathrm{n}=22)$ & $35($ SD 11, $\mathrm{n}=21)$ & $24(\mathrm{n}=1)$ \\
EQ-5D & & $0.68($ SD 0.30, $\mathrm{n}=19)$ & - \\
EQ-5D-VAS & & $74($ SD 20, $\mathrm{n}=19)$ & - \\
VAS pain & $30($ SD 34, range 0-100, $\mathrm{n}=22)$ & $31($ SD: 34, $\mathrm{n}=21)$ & $0(\mathrm{n}=1)$ \\
VAS satisfaction & $77($ SD 30, range 0-100, $\mathrm{n}=22)$ & $81($ SD 25, $\mathrm{n}=21)$ & $0(\mathrm{n}=1)$ \\
\hline
\end{tabular}

HOOS: Hip disability and Osteoarthritis Outcome Score, OHS: Oxford Hip Score, EQ-5D: EuroQol five dimensions questionnaire, VAS: Visual Analogue Score, SD: Standard deviation.

\section{Discussion}

Of 30 included subjects who underwent one-stage revision, 28 were considered successfully treated $(93 \%)$, at a mean follow-up of 40 months. In $50 \%$ of cases, coagulase-negative Staphylococcus was cultured. $84 \%$ of the still living subjects responded to our survey regarding functional outcome and quality of life after one-stage revision for hip PJI.

\section{One-stage revision}

One-stage revision is a treatment option in cases of PJI occurring more than 30 days after initial hip arthroplasty, when there is good soft tissue and bone stock, micro-organism susceptibility to antibiotic agents is high, and bone grafting is not required[2]. Success rates differ, according to selection criteria and study protocols, but lie between 76 and $100 \%$ [4]. See Table 4 for a list of recent and large studies on one-stage revision. The success rate of $93 \%$ that was found in this study is comparable to other studies, in a cohort of subjects with $37 \%$ sinus tracts and $10 \%$ use of allograft bone. The presence of a sinus tract did not seem to lead to worse outcomes in this cohort, as neither of the two patients with treatment failure initially had a sinus tract. In the Infectious Diseases Society of America (IDSA) guidelines, the presence of a sinus tract is no criterion for the choice between one-stage and two-stage revision, although one-stage revision is not recommended when a sinus tract is present[2].

The use of allograft in one-stage revision may have a greater risk of failure according to guidelines[2]. Although the groups are small, both subjects with treatment failure in this cohort underwent one-stage revision with the use of allograft. However, there are also studies that reported good outcomes of one-stage revisions with the use of allograft bone[14,15].

Furthermore, all (3 of 3) micro-organisms in the failure group were multidrug-resistant. On the other hand, $40 \%$ of micro-organisms in the successfully treated group were resistant as well, so successful treatment with one-stage revision, when PJI is caused by multidrug-resistant micro-organisms, is very well possible. However, it may be safe to say that when multidrug-resistant organisms are cultured and bone defects necessitating allograft reconstruction are present, other treatment options, such as two-stage revision, should seriously be considered.

\section{Functional outcome scores}

In this cohort, a HOOS of 63 was found for the successfully treated group. To our knowledge, no studies exist on the HOOS score after one-stage revision or DAIR. We found one study that described a HOOS of 54 after two-stage revision[16]. We found only one other study on HOOS after revision surgery: Mahmoud et al. found a HOOS of around 70 after cup revision with metal augmentation in 147 subjects[17].

We found a mean score of 35 for OHS in this cohort. One other study described postoperative OHS after one-stage revision. They found a score of 31, comparable to the 35 in this study[18]. In other settings, an OHS of 36 after DAIR treatment[19], and an OHS of 32 in 1176 hip revisions (for all reasons)[20] were described. 
Table 4: Overview of studies on one-stage revision.

\begin{tabular}{|c|c|c|c|c|c|c|c|c|}
\hline Author & Year & Country & $\mathrm{N}$ & Success rate & Duration of antibiotic treatment & Functional scores* & QoL scores & Follow-up \\
\hline & \multicolumn{8}{|c|}{ Studies on one-stage only (recent studies with $>20$ cases, all studies with $>100$ cases) } \\
\hline Buchholz & 1981 & Germany & 583 & $77 \%$ & None & - & - & at least 1.5 years \\
\hline Raut & 1995 & UK & 183 & $84 \%$ & 6-12 weeks & $\begin{array}{l}\text { Preop MAP }(9.8) \\
\text { Postop MAP (14.8) }\end{array}$ & - & 93 months \\
\hline Wrobleski & 1986 & UK & 102 & $88 \%$ & up to 6 weeks & - & - & 38 months \\
\hline Vielpeau & 2002 & France & 127 & $88 \%$ & unclear & - & - & 36 months \\
\hline Zeller & 2014 & France & 157 & $94 \%$ & 12 weeks & - & - & 42 months \\
\hline Ilchmann & 2016 & Switzerland & 39 & $100 \%$ & 3 months & Postop HHS (81) & - & 6.6 years \\
\hline Jenny & 2014 & France & 65 & $83 \%$ & 9 weeks (range $0-17$ ) & $\begin{array}{l}\text { Preop MAP (9.5) } \\
\text { Postop MAP (13.3) } \\
\text { Postop OHS (31.0) }\end{array}$ & - & 37 months \\
\hline Whiteside & 2017 & USA & 21 & $95 \%$ & 6 weeks (intra-articular) & - & - & 63 months \\
\hline Winkler & 2008 & Austria & 37 & $92 \%$ & 2 weeks iv, oral not mentioned & - & - & 4.4 years \\
\hline Hansen & 2013 & USA/UK & 27 & $70 \%$ & minimum 6 weeks & - & - & 50 months \\
\hline Bori & 2014 & Spain & 24 & $96 \%$ & not standard, mean 60 days & $\begin{array}{l}\text { Postop MAP (13.8) } \\
\text { Postop HHS (65.4) }\end{array}$ & - & 45 months \\
\hline \multirow[t]{2}{*}{ Rudelli } & 2008 & & 32 & $94 \%$ & 6 months & $\begin{array}{l}\text { Preop MAP (7.0) } \\
\text { Postop MAP (15.9) }\end{array}$ & - & 103 months \\
\hline & \multicolumn{8}{|c|}{ Studies comparing one- and two-stage; results of one-stage group (recent studies with $>10$ cases, all studies with $>100$ cases) } \\
\hline Choi & 2013 & USA & 17 & $82 \%$ & 6 weeks & $\begin{array}{l}\text { Postop HHS (77) } \\
\text { Postop UCLA (4.0) }\end{array}$ & - & 62 months \\
\hline Klouche & 2012 & France & 38 & $100 \%$ & 3 months & $\begin{array}{l}\text { Preop MAP (11.1) } \\
\text { Postop MAP (15.5) }\end{array}$ & - & 35 months \\
\hline Oussedik & 2010 & UK & 11 & $100 \%$ & minimum 6 weeks & $\begin{array}{l}\text { Preop HHS }(40.5) \\
\text { Postop HHS }(87.8 \text {, higher than two stage) } \\
\text { Postop VAS satisfaction ( } 86, \text { higher than two stage) }\end{array}$ & - & 6.8 years \\
\hline De Man & 2011 & Switzerland & 22 & $95 \%$ & 8-12 weeks & Postop HHS (84) & - & 3.8 years \\
\hline
\end{tabular}

Table 4 summarizes other studies that describe functional outcomes after one-stage revision for hip PJI. Most of those studies report outcome using either the Merle d'Aubigné-Postel functional score (MAP) (range 13.3-15.9) or the Harris Hip Score (HHS) (range 65.4-87.8). For both scores, postoperative results were higher than preoperative results. The results of these scores were comparable to results after both two-stage revision and aseptic revision found in other studies[21].

Oussedik et al. found a VAS satisfaction of 86 after one-stage revision, comparable to the 81 in this cohort[5]. This study was one of the four to make a comparison between one-stage and two-stage procedures, but was the only one to find differences in functional scores: VAS satisfaction and HHS after five years were higher after one-stage than after two-stage revision (86 versus 69 and 87.8 versus 75.5, respectively)[5,22-24].

Functional scores between stages in two-stage revision for hip PJI have been reported by Scharferberger et al.[25]. The mean HHS of 54.5 in the interval with a spacer they reported is lower than the HHS after one-stage revision or after aseptic revision. Furthermore, they reported that $56 \%$ of patients were bound to ambulation in their own house.

So, in terms of functional scores, this is the first study to describe HOOS after one-stage revision, and OHS was comparable to one other study on one-stage revision. For both scores, and for other scores described by others, functional outcome of one-stage revision is comparable to aseptic hip revision.
Comparison to two-stage revision shows better outcome after one-stage revision in only one of four studies, but functionality in the interval between stages is lower. Larger and better designed comparable studies are necessary for better comparison.

\section{Quality of life}

The EQ-5D in this cohort was 0.68, and EQ-5D-VAS was 74. In a recent systematic review on QoL after hip PJI treatment, no studies were found on the effect of one-stage revision on quality of life[6]. Also, no other studies on hip PJI were found that used EQ-5D for assessing QoL. However, after prosthetic joint revision surgery in general, two studies found EQ-5D scores of 0.69 and 0.62 , of which Raman et al. found an EQ-5D-VAS of 79[26,27]. These results are similar to the EQ-5D scores found in this study. Both Dawson et al. and Mahmoud et al. found a significant improvement in QoL scores after hip revision surgery[17,27]. Although the QoL after two-stage revision seems comparable to aseptic revision, the QoL in the interval period (without functioning joint) is lower $[6,25]$. We believe one-stage revision improves quality of life for patients comparable to aseptic revision, as no interval period is needed.

\section{Study quality}

Although our study cohort is small, the study setup is retrospective, and it is a single-centre observation, this is the first cohort to describe outcomes of one-stage prosthetic joint replacements in 
terms of quality of life. Unfortunately, no comparison with a comparable cohort after two-stage revision was possible, as only a very small group underwent resection for two-stage revision in our hospital. Also, no scores before revision surgery were available due to the retrospective nature of this study. Furthermore, the group of failures was so small $(n=2)$, that reliable statistical analysis was not possible.

\section{Conclusion}

This study suggests excellent results of one-stage revision, with a success rate of $93 \%$. Functional outcome and quality of life after one-stage revision are both good, and are comparable to prosthetic joint revision surgery in general. Careful and preferably predefined patient selection is required for optimal results. For further research, pre- and postoperative outcome scores are recommended. Studies comparing one-stage revision with DAIR or two-stage revision would be a welcome addition to the current studies.

\section{Competing Interests}

One of the authors performed unpaid consulting services for Debiopharm, received royalties as a book editor from Informa UK ltd., and has been a board member for several Dutch and international expert groups on wound care and diabetic foot infections.

\section{References}

[1] Bozic KJ, Ries MD. The impact of infection after total hip arthroplasty on hospital and surgeon resource utilization. J Bone Joint Surg Am. 2005; 87: 1746-51.

[2] Osmon DR, Berbari EF, Berendt AR, Lew D, Zimmerli W, Steckelberg JM, et al. Diagnosis and management of prosthetic joint infection: Clinical practice guidelines by the infectious Diseases Society of America. Clin Infect Dis. 2013; 56: $1-25$.

[3] Leonard HAC, Liddle AD, Burke O, Murray DW, Pandit H. Single- or two-stage revision for infected total hip arthroplasty? A systematic review of the literature. Clin Orthop Relat Res. 2014; 472: 1036-42.

[4] Nguyen M, Sukeik M, Zahar A, Nizam I, Haddad FS. One-stage Exchange Arthroplasty for Periprosthetic Hip and Knee Joint Infections. Open Orthop J. 2016; 10: 646-53.

[5] Oussedik SIS, Dodd MB, Haddad FS. Outcomes of revision total hip replacement for infection after grading according to a standard protocol. J Bone Joint Surg Br. 2010; 92: 1222-6.

[6] Rietbergen L, Kuiper JWP, Walgrave S, Hak L, Colen S. Quality of life after staged revision for infected total hip arthroplasty: A systematic review. Hip Int. 2016; 26: 311-8.

[7] Strange S, Whitehouse MR, Beswick AD, Board T, Burston A, Burston B, et al. One-stage or two-stage revision surgery for prosthetic hip joint infection - the INFORM trial: a study protocol for a randomised controlled trial. Trials. 2016; 17: 90

[8] Zmistowski B, Della Valle C, Bauer TW, Malizos KN. Diagnosis of Periprosthetic Joint Infection. J Orthop Res. 2014; 32: S98-107.

[9] Magiorakos AP, Srinivasan A, Carey RB, Carmeli Y, Falagas ME, Giske CG, et al. Bacteria : an International Expert Proposal for interim standard definitions for acquired resistance. Microbiology. 2011; 18: 268-81.

[10] Nederlandse Orthopaedische Vereniging (NOV). Patient Reported Outcome Measures. 2012.

[11] Gosens T, Hoefnagels NHM, de Vet RCW, Dhert WJA, van Langelaan EJ, Bulstra SK, et al. The "Oxford Heup Score". Acta Orthop. 2005; 76: 204-11.

[12] de Groot IB, Reijman M, Terwee CB, Bierma-Zeinstra SMA, Favejee M, Roos $\mathrm{EM}$, et al. Validation of the Dutch version of the Hip disability and Osteoarthritis Outcome Score. Osteoarthr Cartil. 2007; 15: 104-9.

[13] Lamers LM, McDonnell J, Stalmeier PFM, Krabbe PFM, Busschbach JJ V. The Dutch tariff: Results and arguments for an effective design for national EQ-5D valuation studies. Health Econ. 2006; 15: 1121-32.
[14] Winkler H, Stoiber a, Kaudela K, Winter F, Menschik F. One stage uncemented revision of infected total hip replacement using cancellous allograft bone impregnated with antibiotics. J Bone Joint Surg Br. 2008; 90: 1580-4.

[15] Loty B, Postel M, Evrard J, Courpied JP. Remplacements en un temps des protheses totales de hanches infectees et reconstructions osseuses par allogreffes. Int Orthop. 1992; 16: 330-8.

[16] van Diemen MPJ, Colen S, Dalemans AAR, Stuyck J, Mulier M. Two-stage revision of an infected total hip arthroplasty: A follow-up of 136 patients. Hip Int. 2013; 23: 445-50.

[17] Mahmoud AN, Sundberg M, Flivik G. Comparable results with porous metal augments in combination with either cemented or uncemented cups in revision hip arthroplasty: an analysis of one hundred forty-seven revisions at a mean of five years. J Arthroplasty. 2017; 32: 1612-7.

[18] Jenny J-Y, Lengert R, Diesinger Y, Gaudias J, Boeri C, Kempf J-F. Routine one-stage exchange for chronic infection after total hip replacement. Int Orthop. 2014; 38: 2477-81.

[19] Grammatopoulos G, Kendrick B, McNally M, Athanasou NA, Atkins B, McLardy-Smith $\mathrm{P}$, et al. Outcome following Debridement, Antibiotics, and Implant Retention in hip Periprosthetic Joint Infection-An 18-Year Experience. J Arthroplasty. 2017; 32: 2248-55.

[20] Philpott A, Weston-Simons JS, Grammatopoulos G, Bejon P, Gill HS, McLardy-Smith $\mathrm{P}$, et al. Predictive outcomes of revision total hip replacement - A consecutive series of 1176 patients with a minimum 10-year follow-up. Maturitas. 2014; 77: 185-90.

[21] Hoberg M, Konrads C, Engelien J, Oschmann D, Holder M, Walcher M, et al. Similar outcomes between two-stage revisions for infection and aseptic hip revisions. Int Orthop. 2016; 40: 459-64.

[22] Choi HR, Kwon YM, Freiberg AA, Malchau H. Comparison of one-stage revision with antibiotic cement versus two-stage revision results for infected total hip arthroplasty. J Arthroplasty. 2013; 28: 66-70.

[23] Klouche S, Leonard P, Zeller V, Lhotellier L, Graff W, Leclerc P, et al. Infected total hip arthroplasty revision: One- or two-stage procedure? Orthop Traumatol Surg Res. 2012; 98: 144-50.

[24] De Man FHR, Sendi P, Zimmerli W, Maurer TB, Ochsner PE, Ilchmann T. Infectiological, functional, and radiographic outcome after revision for prosthetic hip infection according to a strict algorithm. Acta Orthop. 2011; 82: 27-34.

[25] Scharfenberger A, Clark M, Lavoie G, Connor GO, Beaupre LA. Treatment of an infected total hip replacement with the PROSTALAC system. Part 2: Health-related quality of life and function with the PROSTALAC implant in situ. Can J Surg. 2007; 50: 29-33.

[26] Raman R, Kamath RP, Parikh A, Angus PD. Revision of cemented hip arthroplasty using a hydroxyapatite-ceramic-coated femoral component. J Bone Joint Surg Br. 2005; 87: 1061-7.

[27] Dawson J, Fitzpatrick R, Frost S, Gundle R, McLardy-Smith P, Murray D. Evidence for the validity of a patient-based instrument for assessment of outcome after revision hip replacement. J Bone Joint Surg Br. 2001; 83B: $1125-9$ 